\title{
Entity-Based Semantic Adequacy for Data-to-Text Generation
}

\author{
Juliette Faille \\ Université de Lorraine \\ CNRS/LORIA \\ juliette.faille@loria.fr
}

\author{
Albert Gatt \\ Utrecht University \\ a.gatt@uu.nl
}

\author{
Claire Gardent \\ CNRS/LORIA \\ Université de Lorraine \\ claire.gardent@loria.fr
}

\begin{abstract}
While powerful pre-trained language models have improved the fluency of text generation models, it remains difficult to ensure that the generated texts are semantically faithful to the input. In this paper, we introduce a novel automatic evaluation metric, Entity-Based Semantic Adequacy, which can be used to assess to what extent generation models that verbalise RDF (Resource Description Framework) graphs produce text that contains mentions of the entities occurring in the RDF input. This is important as RDF subject and object entities make up $2 / 3$ of the input. We use our metric to compare 25 models from the WebNLG Shared Tasks and we examine correlation with results from human evaluations of semantic adequacy. We show that while our metric correlates with human evaluation scores, this correlation varies with the specifics of the human evaluation setup. This suggests that in order to measure the entity-based adequacy of generated texts, an automatic metric such as the one proposed here might be more reliable, as less subjective and more focused on correct verbalisation of the input, than human evaluation measures.
\end{abstract}

\section{Introduction}

With the introduction of pretrained models, the fluency of text generation systems has improved. However, semantic adequacy (faithfulness to the input) remains an unsolved issue. It remains difficult to ensure that the generated text faithfully captures the input (Wiseman et al., 2017; Gehrmann et al., 2018).

In this paper, we focus on semantic adequacy for RDF-Verbalisers i.e., models such as those submitted to the WebNLG 2017 and 2020 shared tasks (Gardent et al., 2017; Castro Ferreira et al., 2020b) which map an RDF graph to a text verbalising the content of that graph. In this case, the input to Natural Language Generation (NLG) is a set of triples of the form $(e 1, p, e 2)$ where $e 1, e 2$ are RDF entities and $p$ is a property. RDF triplestores are used in particular to model Semantic Web data and their verbalisation aims at making the information from these knowledge-bases easily accessible to users. As exemplified in Figure 1, one necessary condition for the generated text to be semantically adequate is that all entities present in the input should be mentioned at least once in the output. We refer to this requirement as entity-based semantic adequacy (ESA for short). ESA offers one way of formalising the requirement that the output of a generator should reflect the information in the input. Thus, its significance extends beyond the specific problem domain of RDF verbalisation, though the latter provides a useful testcase.

We make the following contributions.

We devise metrics which assess to what extent a text verbalising an RDF graph respects entitybased semantic adequacy. These metrics rely on an algorithm designed to automatically detect whether an entity present in the input graph has a corresponding mention in the output text. We evaluate this algorithm on a corpus of 25,173 (RDF, Text) pairs with manually annotated entity mentions from Castro Ferreira et al. (2018) and show that our algorithm has a recall of 0.74 and a precision of 0.75 .

We apply these metrics to the output of $25 \mathrm{RDF}$ verbalisers developed for the WebNLG 2017 and 2020 challenges and show that some of the systems which rank highest in terms of BLEU scores actually rank in the lower half with respect to entitybased semantic adequacy. This indicates that ESA is measuring a different quality from that measured by surface-based metrics such as BLEU.

We compute correlation between our metrics and both automatic and human evaluation scores collected by the WebNLG organisers for these 25 models. We find a stronger correlation with human metrics related to semantic adequacy than with automatic metrics. Among the automatic metrics, 


\begin{tabular}{|c|c|c|c|c|}
\hline 1 (short) & $\begin{array}{l}\text { Output text } \\
\text { total area of }\end{array}$ & $\begin{array}{l}\text { Liselotte Grschebina is a } \\
20769100000.0 . \\
\text { Liselotte_Grschebina } \\
\text { Israel } \\
\text { Israel } \\
\text { Liselotte_Grschebina } \\
\text { Liselotte_Grschebina }\end{array}$ & $\begin{array}{l}\text { German national w } \\
\text { nationality } \\
\text { areaTotal } \\
\text { officialLanguage } \\
\text { birthPlace } \\
\text { training }\end{array}$ & $\begin{array}{l}\text { Io was born in the German Empire and has a } \\
\text { Israel } \\
20769100000.0 \\
\text { Modern_Standard_Arabic } \\
\text { German_Empire } \\
\text { School_of_Applied_Arts_in_Stuttgart }\end{array}$ \\
\hline 2 (hal) & $\begin{array}{l}\text { Output text } \\
1776 \text {, the col }\end{array}$ & $\begin{array}{l}\text { Born in the Kingdom of } \\
\text { intry is the birth place of } \\
\text { Lady_Anne_Monson } \\
\text { Lady_Anne_Monson } \\
\text { Lady_Anne_Monson } \\
\text { Lady_Anne_Monson } \\
\text { Lady_Anne_Monson }\end{array}$ & $\begin{array}{ll}\text { England in } 1726-0 \\
\text { Joh Davutoglu. } \\
\text { birthPlace } & \text { Darli } \\
\text { birthDate } & 1726 \\
\text { deathDate } & \underline{1776} \\
\text { birthPlace } & \text { King } \\
\text { residence } & \underline{\text { India }}\end{array}$ & $\begin{array}{l}-01 \text {, and living in India, on the 18th of July, } \\
\text { igton } \\
01-01 \\
\frac{02-18}{\text { lom_of_England }}\end{array}$ \\
\hline $3(\mathbf{d e g})$ & $\begin{array}{l}\text { Output tex } \\
\text { the distribu } \\
\text { starred in th }\end{array}$ & $\begin{array}{l}\text { The distributor of the di } \\
\text { on of the distribution of } \\
\text { preparation of the tenni } \\
\text { Super_Capers edit } \\
\text { Super_Capers starn } \\
\text { Super_Capers starn } \\
\text { Super_Capers lang } \\
\text { Super_Capers distril }\end{array}$ & $\begin{array}{ll}\text { tributor of the distr } \\
\text { he distribution of th } \\
\text { Katzman. } \\
\text { ng } & \text { Stacy_Katzl } \\
\text { ng } & \text { Michael_Ro } \\
\text { ng } & \text { Tom_Sizem } \\
\text { age } & \text { English_lan } \\
\text { utor } & \text { Roadside_A }\end{array}$ & $\begin{array}{l}\text { butor of the distributor of the distributor of } \\
\text { e dish, Roadside Attrón, is Tom Botta, who } \\
\text { tan } \\
\text { oker } \\
\text { tre } \\
\text { tractions }\end{array}$ \\
\hline
\end{tabular}

Figure 1: Examples of outputs with low Entity-based Semantic Adequacy. RDF input entities that are missing in the text are underlined (short: the short output fails to mention all input entities, deg: degenerate output, hal: the text hallucinates entities not present in the input and omits to mention others)

correlations are highest with METEOR. Interestingly, we also find that the correlation with human scores varies with the specifics of the human evaluation setup. This suggests that our automatic metric might be a more reliable means of identifying models with low entity-based semantic adequacy than human evaluation. We are publicly releasing our source code. ${ }^{1}$

\section{Related work}

Various methods have been proposed to evaluate the semantic adequacy of generated texts.

Commonly-used metrics are surface-based (either word- or character-based) such as BLEU (Papineni et al., 2002), TER (Snover et al., 2006) or chrF (Popović, 2015). As these methods fail to account for paraphrases, alternative metrics have been proposed such as METEOR (Banerjee and Lavie, 2005), which measures n-gram overlap but integrates synonyms and BERTscore, a trained metric based on word-embeddings similarity (Zhang* et al., 2020). Semantic similarity has also been modeled in terms of propositional content. In computer vision for instance, SPICE transforms both generated and reference captions into a scene graph encoding the objects and relations present in these

\footnotetext{
${ }^{1}$ https://gitlab.nl4xai.eu/juliette. faille/entity-based-semantic-adequacy
}

captions and computes an F-score over the semantic propositions in the scene graph (Anderson et al., 2016). Similarly, the MEANT metric applies Semantic Role Labelling to generated and reference texts and computes similarity by matching the resulting semantic frames. In data-to-text generation, (Dhingra et al., 2019) uses custom entailment models to determine whether an n-gram in the generated text is entailed by the input and computes an F-score based on these n-grams. In text summarisation, Goodrich et al. (2019) compare relation tuples extracted from a ground-truth summary and a generated one using either a Named entity Recognition and a Relation Classifier or an end-to-end Transformer model to extract these tuples.

Rather than abstract over the lexical content of the generated and reference text, other work has focused on developing metrics which model human judgement in particular, judgments of semantic similarity. Thus Sellam et al. (2020) introduced BLEURT, an automatic metric pre-trained on synthetic and automatically rated data and fine-tuned on human judgments.

Closest to our approach are metrics which evaluate the generated output, not with respect to the reference or to human judgments, but with respect to the input. Wiseman et al. (2017) define Relation Generation score as the precision of input relations found in the output texts (the relation ex- 
traction is performed by a neural model). Reed et al. (2018) define information extraction patterns to measure the occurrence of the input attributes and their values in the outputs and compute semantic adequacy using the Slot Error Rate. Ribeiro et al. (2020); Dušek and Kasner (2020) use natural language inference (NLI) to detect two way entailment between the generated text and the input. Sulem et al. (2020) introduce SAMSA which assesses simplification quality by comparing the predicate/argument structures contained in the input with those contained in the output summary.

Similarly, we evaluate the semantic adequacy of a generated text by comparing it with the input. We focus on entities however and provide a detailed assessment of both the reliability of our metrics and its correlation with human and with automatic metrics.

\section{Defining E-Based Semantic Adequacy}

We assume a corpus of $(R, T)$ instances where $R$ is an RDF graph (a set of RDF triples) and $T$ is a text verbalising that graph. RDF triples are of the form $(s, p, o)$ where $p$ is a binary relation holding between a subject $(s)$ and an object $(o)^{2}$. We use the term "entity" to refer to both RDF triple subjects and objects and we write $E_{R}$ for the set of RDF entities occurring in RDF graph $R$.

Entity Mentions. Given a corpus instance $(R, T)$, an entity mention $m$ is a text segment in $T$ which denotes an entity $e$ present in the input graph $(e \in$ $\left.E_{R}\right)$. We write $M_{T}$ for the set of entity mentions occurring in $T$ and $\llbracket m \rrbracket=e$ to indicate that the mention $m \in T$ denotes entity $e \in E_{R}$.

(Un)Detected Entities. A detected entity $e \in$ $E_{R}$ is an entity which has a matching mention in $M_{T}$ i.e., there is a mention $m \in M_{T}$ such that $\llbracket m \rrbracket=e$. Conversely, an undetected entity is an entity $e \in E_{R}$ which has no corresponding mention in $M_{T}$. We define $E_{T} \subseteq E_{R}$ as the set of RDF entities which have a corresponding mention in $T$.

Entity-Based Semantic Adequacy. Given an $(R, T)$ pair, we define entity-based semantic adequacy (ESA) as the proportion of RDF entities in $E_{R}$ which have a corresponding mention $m \in M_{T}$. In other words, ESA is the ratio between the number of entities for which a mention was found $\left(E_{T}\right)$

\footnotetext{
${ }^{2}$ Subjects are Uniform Resource Identifiers (URI) and objects are either URIs or literals. Intuitively, RDF subjects and objects refer to things such as persons, locations, abstract entities, dates or phone numbers.
}

and the total number of entities occurring in the input $\operatorname{RDF}\left(E_{R}\right)$.

$$
\operatorname{ESA}_{I}=\frac{\left|E_{T}\right|}{\left|E_{R}\right|}
$$

Given a corpus of $(R, T)$ pairs, we also compute the proportion of texts in that corpus with at least $n$ undetected entities. We refer to this metric as corpus-level, entity-based semantic inadequacy at $n$ (ESI ${ }^{n}$ for short).

\section{Computing E-Based Semantic Adequacy}

The metrics introduced in the previous section rely on being able to determine which entities in the RDF input have a matching mention in the corresponding text. We present an algorithm for entity mention detection and we report on an evaluation of that algorithm using a dataset of 25,173 (RDF graph, Text) pairs where entity mentions have been manually annotated.

\subsection{Detecting Entity Mentions}

We define our entity mention detection algorithm using a combination of existing tools and heuristics.

Entity linker We use the state-of-the-art REL entity linker from van Hulst et al. (2020). When applied to a text, REL returns a list of entity mentions and their corresponding DBPedia entities. We filter out the mentions for which the related DBPedia entity does not match any of the input RDF entities.

Approximate string matching of text $n$-grams and RDF entities We match text n-grams to candidate RDF entities, using approximate string matching with a fixed maximum allowed edit distance (normalized Levenshtein distance). This value is experimentally fixed at $0.4^{3}$. To improve results, we create a dictionary of RDF entity synonyms and compute the approximate match between text n-grams and all RDF entities, including their synonyms. The synonym dictionary was initially created using DBPedia aliases and rules (handcrafted to improve the detection of frequent entities in the WebNLG corpus such as places or quantities). During the evaluation of the entity detection (cf section 4.2), an expanded version of

\footnotetext{
${ }^{3}$ The algorithm used by our string matching procedure is described in detail in Algorithm 1 in the Appendix.
} 
the dictionary was developed by updating it with entities for which no mention was detected in an evaluated text; these were manually included in the dictionary. This dictionary provides a symbolic means to improve entity mention detection and more generally, to adapt the algorithm to a new domain. However, it should be noted that, on the WebNLG 2017 dataset, adding this dictionary only slightly improves entity mention detection and is not essential.

Pronominal entity mentions In order to detect which input entity a pronoun refers to, we use two methods. We first use Stanza (Qi et al., 2020) to compute co-reference chains in our texts, keeping only the pronominal mentions. For the pronouns that were not detected by the previous method, we used a simple heuristic. In the WebNLG corpus, RDF graphs are created with a single entity as "root". Other entities are meant to describe and provide information about this root. As the texts are quite short we assume that most pronominal anaphors refer to the "root" of the graph. We therefore associate all remaining pronouns to the root entity of the RDF graph.

Dates We use the python library dateparser to normalise dates both in the text and in the RDF. Results are further filtered using entity type information from the input RDF graph.

Putting it all together. Each method described above yields a list of mentions found in a text for each RDF entity in an input graph. In case different methods identify the same (or overlapping) mentions, we select those mentions with the lowest edit distance to their matched RDF entity (or one of its synonyms). In case of equality, we keep the longest mention.

\subsection{Evaluating automatic entity mention detection}

Castro Ferreira et al. (2018) manually annotated entity mentions in the WebNLG 2017 dataset (an example of annotation is shown in the Appendix). We use these manual annotations as gold standard to evaluate our entity mentions detection algorithm. Given $M^{\text {auto }}$, the set of mentions detected on this corpus by our mention detection algorithm and $M^{\text {human }}$, the set of manually annotated mentions, we compute Recall and Precision in the usual way: Recall $=\frac{\left|M^{\text {auto }} \cap M^{\text {human }}\right|}{\left|M^{\text {human }}\right|}$ and
Precision $=\frac{\left|M^{\text {auto }} \cap M^{\text {human }}\right|}{\left|M^{\text {auto }}\right|}$. The intersection $M^{\text {auto }} \cap M^{\text {human }}$ is the number of exact string matches between the sets of mentions $M^{\text {auto }}$ and $M^{\text {human }}$. We obtain a recall of 0.74 and a precision of 0.75 .

If we consider approximate string matching in the computation of $M^{\text {auto }} \cap M^{\text {human }}$ with a maximum allowed normalized edit distance of 0.2 , we obtain a recall of 0.82 and a precision of 0.83 . This shows that although some of the automatically detected mentions do not match the gold standard annotations exactly, they are nonetheless close to them. In Section 5.2 below, we show that even though imperfect, our entity mention detection algorithm permits reliably identifying models which have low entity-based semantic adequacy.

\section{Evaluating RDF-to-text Generation Models}

25 models participated in the WebNLG 2017 and 2020 challenges. We apply our entity-based semantic adequacy metrics to the output of these models on the WebNLG 2017 and 2020 test data ${ }^{4}$. We group models with respect to BLEU and $\mathrm{ESI}_{C}{ }^{1}$ rank. As the text output by the models might differ from the crowdsourced texts we used for the evaluation presented in Section 4.2, we report on a manual verification of our entity mention detection algorithm on a sample from these model outputs. Finally, we show some example outputs illustrating different ways in which a generated text might have low entity-based semantic adequacy.

\subsection{Entity-Based Semantic Adequacy in the WebNLG Shared Tasks}

For each model in the WebNLG 2017 and 2020 Shared Tasks, we compute the $\mathrm{ESI}_{C}{ }^{1}$ score (proportion of texts with one or more RDF entities lacking a matching text mention) and the ESA $\mathrm{E}_{I}$ score (proportion of RDF entities in the input with a matching entity mention in the output). The $\mathrm{ESA}_{I}$ scores are averaged over the corpus in three different ways, over all texts (ESA $C$ score), texts that have at least one undetected entity $\left(\mathrm{ESA}_{C} \backslash 1\right)$ and texts with at least two undetected entities ( $\mathrm{ESA}_{C} \backslash 2$ ). Table 1 shows the results together with the distribution of undetected entities.

\footnotetext{
${ }^{4}$ Examples of outputs of the entity mentions detection are given in the Appendix.
} 


\begin{tabular}{|c|c|}
\hline & Model= NUIG-DSI, BLEU $=41.33$, ESA $_{I}=0.63$ \\
\hline Text & $\begin{array}{l}\text { The record label of Bootleg Series Volume 1: The Quine Tapes is Polydor Records and it was recorded } \\
\text { in St Louis, Missouri, United States. The album was preceded by Squeeze (The Velvet Underground } \\
\text { album). }\end{array}$ \\
\hline \multirow[t]{7}{*}{ RDF Input } & Bootleg Series Volume 1: The Quine Tapes I recordedIn I United States \\
\hline & Bootleg Series Volume 1: The Quine Tapes I recordedIn | St. Louis, Missouri \\
\hline & Bootleg_Series_Volume_1:_The_Quine_Tapes | precededBy I Squeeze_(The_Velvet_Underground_album) \\
\hline & Bootleg_Series_Volume_1:_The_Quine_Tapes | recordLabel | Polydor_Records \\
\hline & Bootleg_Series_Volume_1:_The_Quine_Tapes | recordLabel | Universal_Music_Group \\
\hline & Bootleg_Series_Volume_1:_The_Quine_Tapes | releaseDate | $2001-10-16$ \\
\hline & Bootleg_Series_Volume_1:_The_Quine_Tapes | runtime | 230.05 \\
\hline Text & $\begin{array}{l}\text { Model }=\text { CycleGT, } \mathbf{B L E U}=\mathbf{4 4 . 5 9}, \mathbf{E S A}_{I}=\mathbf{0 . 7 5} \\
\text { the } 11 \text { th Mississippi Infantry Monument was established in } 2000 \text { and is located in Cumberland County, } \\
\text { Pennsylvania. }\end{array}$ \\
\hline \multirow[t]{3}{*}{ RDF Input } & 11th_Mississippi_Infantry_Monument I established | 2000 \\
\hline & 11th_Mississippi_Infantry_Monument | location I Adams_County,_Pennsylvania \\
\hline & Adams_County,_Pennsylvania | hasToItsNorth | Cumberland_County,_Pennsylvania \\
\hline & Model= NUIG-DSI, BLEU $=47.92, \mathrm{ESA}_{I}=0.67$ \\
\hline Text & $\begin{array}{l}\text { The Acharya Institute of Technology is located in Soldevanahalli, Acharya Dr. Sarvapalli Radhakrishnan } \\
\text { Road, Hessarghatta Main Road, Bangalore - 560090. Its director is Dr. G. P. Prabhukumar and it is } \\
\text { located in Mumbai. }\end{array}$ \\
\hline \multirow[t]{6}{*}{ RDF Input } & $\begin{array}{l}\text { Acharya_Institute_of_Technology I campus I "In Soldevanahalli, Acharya Dr. Sarvapalli Radhakrishnan } \\
\text { Road, Hessarghatta Main Road, Bangalore - 560090." }\end{array}$ \\
\hline & All_India_Council_for_Technical_Education I location I Mumbai \\
\hline & Acharya_Institute_of_Technology I director | "Dr. G. P. Prabhukumar" \\
\hline & a_Institute_of_Technology | city | Bangalore \\
\hline & Acharya_Institute_of_Technology l \\
\hline & All India Council for Technical Education \\
\hline
\end{tabular}

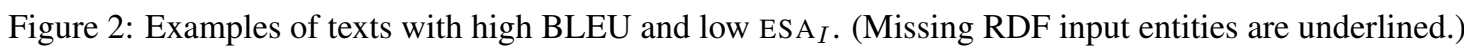

2017 vs. 2020. We see a marked improvement between 2017 and 2020. While in 2017, the ratio of generated texts failing to mention at least one entity varies from 10 to $77 \%$ whereas in 2020 it ranges between $3 \%$ and $51 \%$. The trend is similar for the various $\mathrm{ESA}_{C}$ scores with e.g., an $\mathrm{ESA}_{C} \backslash 2$ range of $[0.17,0.64]$ in 2017 against [0.36,0.71] in 2020. This corroborates the impression that Natural Language Generation (NLG) models have strongly improved in recent years.

2020 NLG. Zooming in on the more state-ofthe-art 2020 models, we find that out of a total of 1779 texts and 16 model outputs, the average $\mathrm{ESI}_{C}{ }^{1}$ score is $17 \%$ and the median $10 \%$. In other words, on average, models fail to mention at least one entity $17 \%$ of the time.

There are strong differences between the models however. The rule-based models (RALI, Baseline2017, DANGNT-SGU, Baseline-2020) have low $\mathrm{ESI}_{C}{ }^{1}$. This is unsurprising as such models can integrate lexicons mapping RDF entities to natural language mentions. Interestingly, among the other five models with an $\mathrm{ESI}_{C}{ }^{1}$ less than $11 \%$, four are bilingual neural NLG models i.e., models which were trained to transform RDF data not only in English but also in Russian.
High BLEU does not guarantee Entity-Based Semantic Adequacy. Figure 3 clusters models with respect to both BLEU and $\mathrm{ESI}_{C}{ }^{1}$ ranks. Models that occur right of the vertical axis have high ${ }^{E S I} C$ rank (they are in the first 8 group), models that occur above the horizontal axis have high BLEU rank. We see that from the 8 models with highest BLEU rank, only three are also among the 8 models with highest $\mathrm{ESI}_{C}{ }^{1}$ rank (cuni-ufal, FBConvAI and Amazon_AI). The five other models which rank among the first eight in terms of BLEU score (OSU, CycleGT, NUIG, TGen, bt5) have a BLEU score ranging between 0.45 and 0.54 yet their $\mathrm{ESI}_{C}{ }^{1}$ score ranges between 10 and $22 \%$. This highlights the fact that a high BLEU score does not guarantee semantic adequacy: while their BLEU score is high, on average these models fail to mention at least one of the input entities 10 to $22 \%$ of the time. Figure 2 shows some examples of 2020 outputs with low ESA $I$ and high BLEU score.

Figure 3 further shows that no model ranks high in term of both BLEU and entity-based semantic adequacy (no model in the top right corner).

\subsection{Manual Verification of the $\operatorname{ESI}_{C}$ results}

Our mention detection algorithm does not detect all mentions, while texts generated by the WebNLG 


\begin{tabular}{|c|c|c|c|c|c|c|c|c|c|c|c|c|}
\hline Model & 1 & 2 & 3 & 4 & $5-8$ & $>1$ & $\downarrow \mathrm{ESI}_{C}{ }^{1}$ & Type & BLEU & $\uparrow \mathrm{ESA}_{C}$ & $\uparrow \mathrm{ESA}_{C} \backslash 1$ & $\uparrow \mathrm{ESA}_{C} \backslash 2$ \\
\hline \multicolumn{13}{|l|}{2020} \\
\hline RALI & 50 & 1 & & & 0 & 51 & $3 \%$ & Symb & 11 & 0.99 & 0.8 & 0.6 \\
\hline Baseline-2020 & 55 & 1 & & & 0 & 56 & $3 \%$ & Symb & 10 & 0.99 & 0.78 & 0.6 \\
\hline Huawei & 62 & 4 & & & 0 & 66 & $4 \%$ & T5 & 12 & 0.99 & 0.79 & 0.65 \\
\hline DANGNT-SGU & 98 & 2 & 1 & & 0 & 101 & $6 \%$ & Symb & 9 & 0.99 & 0.76 & 0.56 \\
\hline Baseline-2017 & 94 & 27 & 0 & 2 & 7 & 130 & $7 \%$ & Symb & 15 & 0.97 & 0.65 & 0.36 \\
\hline FBConvAI & 154 & 4 & & & 0 & 158 & $9 \%$ & BART & 3 & 0.98 & 0.77 & 0.62 \\
\hline cuni-ufal & 169 & 11 & 2 & & 0 & 182 & $10 \%$ & mBART & 7 & 0.98 & 0.78 & 0.65 \\
\hline Amazon_AI & 175 & 10 & & & 0 & 185 & $10 \%$ & T5 & 1 & 0.98 & 0.78 & 0.69 \\
\hline OSU & 171 & 13 & 1 & & 0 & 185 & $10 \%$ & T5 & 2 & 0.98 & 0.78 & 0.65 \\
\hline CycleGT & 240 & 25 & 1 & & 0 & 266 & $15 \%$ & T5 & 8 & 0.97 & 0.81 & 0.71 \\
\hline NUIG-DSI & 203 & 62 & 17 & 0 & 1 & 283 & $16 \%$ & T5 & 4 & 0.96 & 0.76 & 0.67 \\
\hline bt5 & 305 & 45 & 4 & & 0 & 354 & $20 \%$ & T5 & 5 & 0.95 & 0.76 & 0.62 \\
\hline TGen & 264 & 78 & 26 & 15 & 17 & 400 & $22 \%$ & T5 & 6 & 0.94 & 0.72 & 0.58 \\
\hline NILC & 499 & 123 & 13 & 5 & 0 & 640 & $36 \%$ & BART & 16 & 0.89 & 0.69 & 0.56 \\
\hline ORANGE & 600 & 190 & 45 & 9 & 2 & 846 & $48 \%$ & BART & 14 & 0.83 & 0.65 & 0.5 \\
\hline UPC-POE & 589 & 230 & 63 & 19 & 7 & 908 & $51 \%$ & T5 & 13 & 0.84 & 0.7 & 0.59 \\
\hline \multicolumn{13}{|l|}{2017} \\
\hline Tilburg SMT & 179 & 8 & & & & 187 & $10 \%$ & SMT & 2 & 0.97 & 0.7 & 0.55 \\
\hline UPF-FORGe & 203 & 18 & & & & 221 & $12 \%$ & Symb & 4 & 0.97 & 0.73 & 0.56 \\
\hline Melbourne & 371 & 74 & 11 & & & 456 & $24 \%$ & NMT & 1 & 0.94 & 0.76 & 0.64 \\
\hline Tilburg NMT & 555 & 171 & 20 & 3 & & 749 & $40 \%$ & NMT & 6 & 0.89 & 0.72 & 0.62 \\
\hline Tilburg Pipeline & 304 & 233 & 122 & 72 & 49 & 780 & $42 \%$ & Symb & 5 & 0.76 & 0.42 & 0.2 \\
\hline Adapt & 482 & 295 & 130 & 52 & 15 & 974 & $52 \%$ & NMT & 8 & 0.76 & 0.54 & 0.38 \\
\hline PKUWriter & 529 & 282 & 135 & 106 & 60 & 1112 & $60 \%$ & NMT & 3 & 0.71 & 0.52 & 0.36 \\
\hline UIT-DANGNT & 47 & 138 & 238 & 317 & 630 & 1370 & $74 \%$ & Symb & 9 & 0.28 & 0.02 & 0 \\
\hline Baseline & 377 & 398 & 249 & 207 & 206 & 1437 & $77 \%$ & NMT & 7 & 0.47 & 0.31 & 0.17 \\
\hline
\end{tabular}

Table 1: Entity-Based Semantic Adequacy of the WebNLG Challenge 2020 and 2017 Participant Models. ESI ${ }_{C}^{1}$ : Proportion of texts with at least one undetected mention (lower is better). The second to sixth columns indicate the number of texts with $n$ undetected entities. The last three columns give the corpus average of the text level $\mathrm{ESA}_{I}$ score, for all texts $\left(\mathrm{ESA}_{C}\right)$, for texts with at least one undetected entity $\left(\mathrm{ESA}_{C} \backslash 1\right)$ and for texts with at least two undetected entities (ESA $\backslash 1$ ). For ESA $I$ scores, higher is better. BLEU indicates the rank of the model in terms of BLEU in the WebNLG Shared Task and Type, the type of model (Symb: the model integrates a symbolic component, BART, mBART, T5: the pre-trained model used).

models might differ from the crowdsourced texts on which we evaluated our entity mention detection algorithm (cf. Section 4.2). Therefore, we manually verify the result of our mentions detection algorithm for different types of models. We focus on five models with contrasting BLEU and $\mathrm{ESI}_{C}{ }^{1}$ rank, two models with high $\mathrm{ESI}_{C}{ }^{1}$ rank but low BLEU rank; one model with high rank for both dimensions; one model with high BLEU rank and low $\mathrm{ESI}_{C}{ }^{1}$ rank; and one model with low rank in both dimensions. For each of these models, we check texts with different numbers of missing entities (one or two missing entities for the models with high $\mathrm{ESI}_{C}{ }^{1}$ and one, three and five missing entities for the models with low $\operatorname{ESI}_{C}{ }^{1}$ ) and computed the rate of false positives, i.e. entities which were labeled as undetected by our algorithm but which are in fact present in the generated text. While for the three models which rank high in terms of entitybased semantic adequacy, the rate of false positive is high (100\% for RALI, $81 \%$ for Huawei and $52 \%$ for $\mathrm{FBConvAI}^{5}$, for models with low $\mathrm{ESI}_{C}$ rank, the number of false positives is much lower (49\% for bt5, 13\% for Orange). In other words, our entity mention detection algorithm is best at detecting models with low semantic adequacy.

\subsection{Qualitative Analysis}

Examining outputs with low ESA score, we found three main causes for low semantic adequacy: short output, hallucination and degenerate output. Figure 1 shows some examples. When the output text is much shorter than expected, many mentions are missing (Ex.1). When the model hallucinates entities not present in the input, it also often simultaneously fails to mention those that are (Ex. 2).

\footnotetext{
${ }^{5}$ The repetition of the same entities is different entries of the dataset has a strong impact here. For instance, for the RALI system, on the 1779 texts we checked, our algorithm finds 51 texts with undetected entities but only nine of these entities are distinct. That is, the algorithm fails to detect nine entities and as these are in multiple corpus instances, it has $100 \%$ of false positives on these corpus instances.
} 


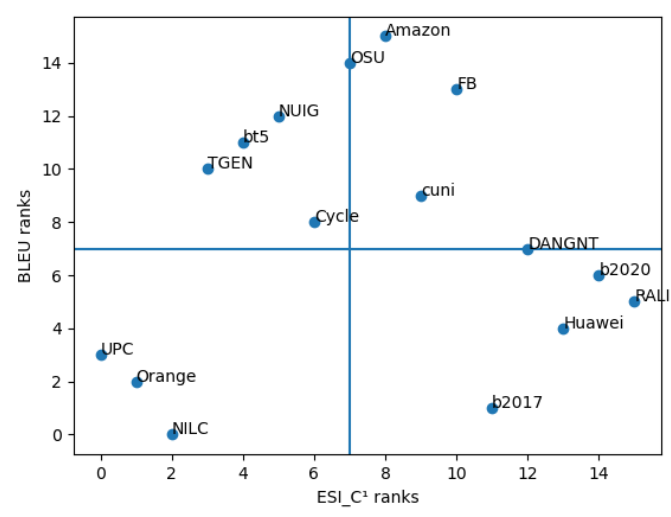

Figure 3: BLEU vs ESI $_{\mathbf{C}}^{1}$ ranks for WebNLG2020 models (higher is better i.e., position 8 in the graph indicates the highest ranked system). The top part of the figure shows the top 8 ranked models w.r.t. BLEU, the right most part the top 8 ranked models w.r.t. ESA. Of the 8 top ranked models w.r.t. BLEU (top part of the figure), only two (FB and OSU) are among the 8 top ranked w.r.t. ESA scores.

Finally, entity mentions may be missing because of degenerate output (Ex.3).

\section{Correlation with Human and Automatic Metrics}

We study the correlation of our ESA $I$ metric with the human and automatic metrics used in the WebNLG challenges.

\subsection{Evaluation Set-Up}

During the WebNLG Challenges 2017 and 2020, 223 texts were sampled from the outputs of the participants models for human evaluation in 2017, and 178 in 2020 (Shimorina et al., 2018; Castro Ferreira et al., 2020a). For our correlation study, we therefore use the automatic and human evaluation scores collected by the WebNLG organisers for 2,007 texts ( 223 for each of the 9 models) in 2017 and 2,848 texts (178 for each of the 16 models) in 2020. We use the results and scripts from Shimorina et al. (2018) and Castro Ferreira et al. (2020a).

In 2017, the human evaluation metric which focuses on semantic adequacy is Semantics where the annotator is asked to assess semantic faithfulness of the generated output w.r.t. the input (1-low, 2 -medium or 3-good). In 2020, the human evaluation metrics concerned with semantic adequacy are Data Coverage, Correctness, Relevance (between 0 and 100). For Data Coverage, evaluators were asked to check whether all input RDF properties were in the text; for Relevance, whether the text describes only such predicates which were present in the input; and for Correctness, whether the output text correctly describes the subject and object of those predicates which matched a property in the input graph. Note that while all these criteria bear on semantic adequacy, none of them specifically target entities.

\subsection{Results}

We compute the correlations with $\mathrm{ESA}_{I}$ metric at text level in three different set-ups (for all texts, for texts with at least one undetected entity and for texts with at least two undetected entities) using three correlation metrics (Pearson correlation, the Spearman rank correlation and Kendall's Tau). Tables 2 and 3 only report Pearson correlations on texts with at least one undetected entity $(n=822$ for 2017; $n=470$ for 2020). Detailed correlation results for the three metrics and considering the three text setups are reported in the Appendix.

Human vs. Automatic Metrics. The correlation between $\mathrm{ESA}_{I}$ and human metrics of semantic adequacy is strong in 2017 and moderate in 2020, indicating that $\mathrm{ESA}_{I}$ correctly captures what humans judge to be semantically adequate. ESA also has very strong (2017) and moderate (2020) correlation with METEOR, which suggests that variants of entity mentions involve synonyms and stemming like modifications.

Varying Correlation Strengths and Scale. The strength of the correlations and their relative order vary with the shared tasks. For automatic metrics, this is likely due to greater variance in metric scores between systems. For human judgments, it might also result from the different criteria used in 2017 vs. 2020 and from their subjectivity. Indeed, as shown below, some of the collected human judgments are in fact incorrect. Not shown here, but reported in the appendix, correlations with $\mathrm{Hu}$ man, METEOR and BLEU scores also tend to be higher for texts with at least one or two undetected entities - that is, texts which are likely to have semantic adequacy problems - compared to correlations computed over all texts (compare Table 3 to correlations over all texts, in the Appendix).

Cases of strong disagreement between $\operatorname{ESA}_{I}$ and human evaluation We manually checked some of the texts which received high human evaluation scores but had a low proportion of detected 


\begin{tabular}{l|llllll} 
Metrics & METEOR & TER & Fluency & Grammar & Semantics & ESA $_{I}$ \\
\hline BLEU & 0.74 & -0.57 & 0.39 & 0.43 & 0.53 & 0.59 \\
METEOR & $\mathrm{x}$ & -0.54 & 0.57 & 0.63 & 0.72 & $\mathbf{0 . 8 7}$ \\
TER & $\mathrm{x}$ & $\mathrm{x}$ & -0.42 & -0.45 & -0.4 & -0.42 \\
\hline Fluency & $\mathrm{x}$ & $\mathrm{x}$ & $\mathrm{x}$ & 0.89 & 0.51 & 0.49 \\
Grammar & $\mathrm{x}$ & $\mathrm{x}$ & $\mathrm{x}$ & $\mathrm{x}$ & 0.57 & 0.57 \\
Semantics & $\mathrm{x}$ & $\mathrm{x}$ & $\mathrm{x}$ & $\mathrm{x}$ & $\mathrm{x}$ & $\mathbf{0 . 6 6}$
\end{tabular}

Table 2: Pearson correlation coefficients for WebNLG 2017 metrics and ESA $A_{I}$. Only for texts with at least one undetected entity (i.e. 822 texts). All the p-values are $<0.01$. Bold numbers indicate the highest correlations of ESA $_{I}$ with surface-based (top block) and human evaluation (bottom block) metrics.

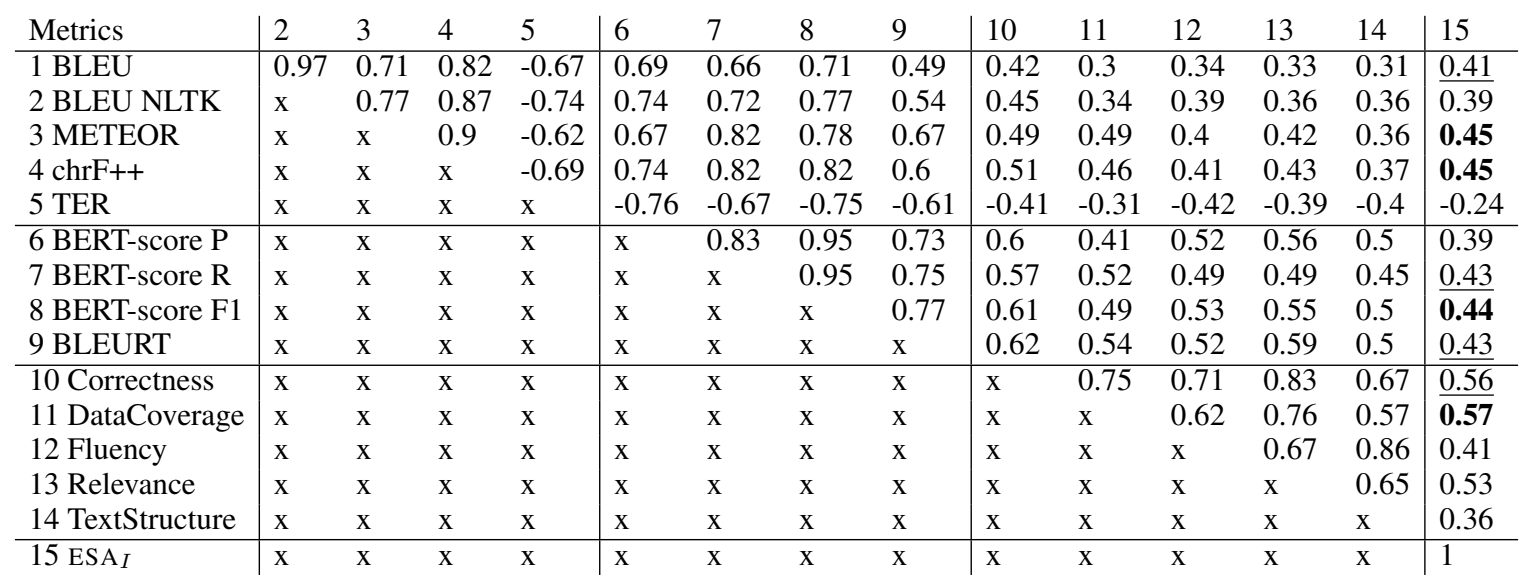

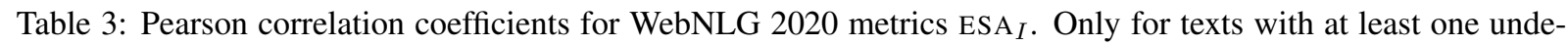
tected entity (i.e. 470 texts). All the p-values are $<0.01$. Bold (resp. underlined) numbers indicate the highest (resp. second highest) correlation scores between $\mathrm{ESA}_{I}$ and different categories of evaluation metrics, i.e. surface-based similarity metrics (top block), embedding-based similarity (middle block) and human evaluation metrics.

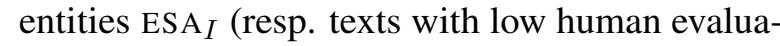
tion scores but high $\mathrm{ESA}_{I}$ ). ${ }^{6}$

For WebNLG 2017, there are 7 texts that have ESA $_{I}<0.4$ and Semantics $\geq 2$. For 6 of them we find that there are indeed missing entities, while the remaining one is a degenerate text. The relatively high scores given by human evaluators for Semantics (2 out of 3 ) suggest that such scoring tends to be subjective, perhaps especially so with a broad evaluation criterion such as 'Semantics'. Among the 41 texts which received the lowest possible rating for semantics (Semantics=1), but had $\mathrm{ESA}_{I}>0.9$, we find that 25 texts do not have missing entities but are indeed semantically incorrect (usually because of mistakes or hallucinations of predicates); 3 texts have missing entities and 9 texts have hallucinated entities. The remaining 4 texts contain all input entities and have correct semantics.

The same kind of observations can be made for WebNLG 2020 models. There are 4 texts which received high human evaluation scores for semantic adequacy-related criteria (Data Coverage $>80$

\footnotetext{
${ }^{6}$ Examples are given in the appendix.
}

or Correctness $>80$ or Relevance $>80$ ) and low $\mathrm{ESA}_{I}\left(\mathrm{ESA}_{I}<0.4\right)$ and all of them have missing entities. In contrast, there are 20 texts which got low human evaluation scores (Data Coverage $<30$ or Correctness $<30$ or Relevance $<30$ ) and high $\mathrm{ESA}_{I}\left(\mathrm{ESA}_{I}>0.9\right)$. Fifteen have wrong or hallucinated predicates, two have significant spelling or repetition problems. Three texts are correct.

From these observations we can draw two main conclusions. First, detecting input RDF entities in the output text is no sufficient condition to assess a model's semantic adequacy. It does not give information about hallucination of entities or about correct verbalization of RDF predicates, which are also necessary conditions for semantic adequacy. These observations also illustrate the subjectivity of human evaluation. Sometimes correct texts can be rated badly by human annotators or vice versa.

Detection of Hallucinations We can use our entity mention detection algorithm in reverse to detect hallucinations i.e., mentions that have no corresponding RDF entity in the input graph. We gather all (entity, mention) pairs found by the entity linker (4.1) for which the entity does not occur in the in- 


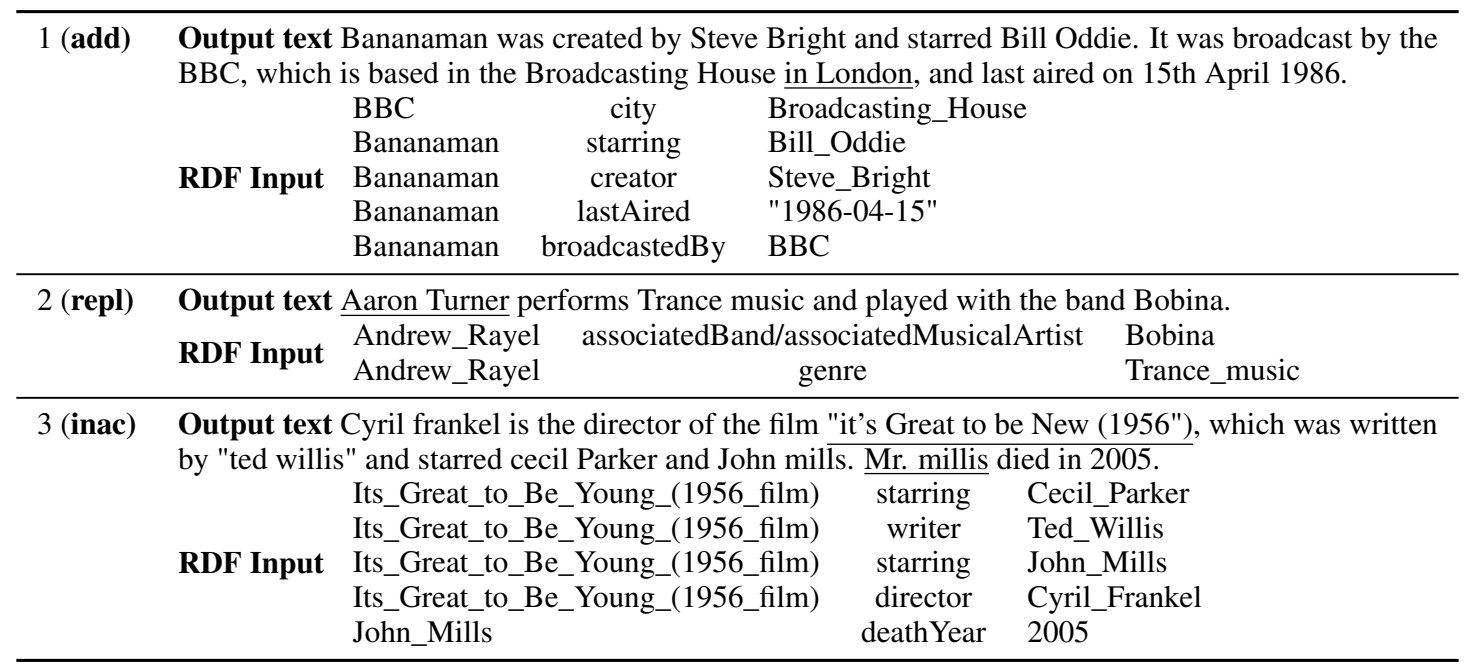

Figure 4: Examples of hallucinations (underlined in the texts). add: output contains additional information, repl: an input RDF entity is replaced by another with the same context (here the name of another musician), inac: the name of the input entities are inaccurate which makes them difficult to link with input entities

\begin{tabular}{l|llll} 
Model & $>1$ & $>1 \checkmark$ & Dist & $\downarrow$ ESI $_{C}{ }^{1}$ \\
\hline RALI & 0 & 0 & 0 & $0 \%$ \\
B-2017 & 1 & 1 & 1 & $0.1 \%$ \\
B-2020 & 1 & 1 & 1 & $0.1 \%$ \\
NUIG & 4 & 3 & 3 & $0.2 \%$ \\
UPC & 4 & 4 & 3 & $0.2 \%$ \\
DANGNT & 5 & 5 & 5 & $0.3 \%$ \\
TGen & 8 & 7 & 2 & $0.5 \%$ \\
cuni-ufal & 9 & 7 & 6 & $0.5 \%$ \\
Amazon & 9 & 9 & 3 & $0.5 \%$ \\
FBConvAI & 17 & 11 & 6 & $1 \%$ \\
CycleGT & 19 & 18 & 10 & $1 \%$ \\
OSU & 20 & 19 & 3 & $1 \%$ \\
bt5 & 36 & 17 & 3 & $2 \%$ \\
Huawei & 48 & 47 & 28 & $3 \%$ \\
NILC & 117 & 99 & 66 & $7 \%$ \\
ORANGE & 288 & 288 & 60 & $16 \%$ \\
\hline UIT & 1 & 0 & 1 & $0.1 \%$ \\
Tilburg SMT & 4 & 0 & 4 & $0.2 \%$ \\
Tilburg NMT & 11 & 4 & 7 & $0.6 \%$ \\
UPF & 12 & 8 & 4 & $0.6 \%$ \\
Tilburg Pl & 14 & 11 & 6 & $0.8 \%$ \\
Melbourne & 114 & 112 & 24 & $6 \%$ \\
Adapt & 241 & 234 & 151 & $13 \%$ \\
PKUWriter & 286 & 283 & 135 & $15 \%$ \\
Baseline & 754 & 144 & 147 & $40 \%$
\end{tabular}

Table 4: Hallucinations ( $>1$ and $>1_{\checkmark}$ : number of texts with at least one hallucination before and after a manual check of automatically detected hallucinations. *Verification on 144 randomly chosen texts. Dist: number of distinct detected hallucinated entities. put RDF graph. Table 4 summarizes the results for each model of the WebNLG challenges. Figure 4 also shows examples of different types of hallucinations.

\section{Conclusion}

RDF stores have become increasingly popular as a means to make knowledge available on the web (Assi et al., 2020). We propose an automatic metric for assessing the entity-based semantic adequacy of RDF verbalisers and show that it is effective in highlighting semantic inadequacy even for state-ofthe-art models with high BLEU scores. We further show that models detected by this metric as having low entity-based semantic adequacy can still have high scores on surface-based metrics, and that while ESA correlates with human scores on semantic criteria, it may in fact be more reliable as a means of detecting low performing models than human-based evaluation protocols, which tend to be subjective.

\section{Acknowledgments}

Research reported in this paper has received funding from the European Union's Horizon 2020 research and innovation programme under the Marie Skłodowska-Curie grant agreement No 860621 . This document reflects the views of the authors and does not necessarily reflect the views or policy of the European Com-mission. The REA cannot be held responsible for any use that may be made of the information this document contains. 


\section{References}

Peter Anderson, Basura Fernando, Mark Johnson, and Stephen Gould. 2016. Spice: Semantic propositional image caption evaluation. In European conference on computer vision, pages 382-398. Springer.

Ali Assi, Hamid Mcheick, and Wajdi Dhifli. 2020. Data linking over rdf knowledge graphs: A survey. Concurrency and Computation: Practice and Experience, 32(19): 5746.

Satanjeev Banerjee and Alon Lavie. 2005. METEOR: An automatic metric for MT evaluation with improved correlation with human judgments. In Proceedings of the ACL Workshop on Intrinsic and Extrinsic Evaluation Measures for Machine Translation and/or Summarization, pages 65-72, Ann Arbor, Michigan. Association for Computational Linguistics.

Thiago Castro Ferreira, Claire Gardent, Nikolai Ilinykh, Chris van der Lee, Simon Mille, Diego Moussallem, and Anastasia Shimorina. 2020a. The 2020 bilingual, bi-directional WebNLG+ shared task: Overview and evaluation results (WebNLG+ 2020). In Proceedings of the 3rd International Workshop on Natural Language Generation from the Semantic Web (WebNLG+), pages 55-76, Dublin, Ireland (Virtual). Association for Computational Linguistics.

Thiago Castro Ferreira, Claire Gardent, Nikolai Ilinykh, Chris van der Lee, Simon Mille, Diego Moussallem, and Anastasia Shimorina, editors. 2020b. Proceedings of the 3rd International Workshop on Natural Language Generation from the Semantic Web (WebNLG+). Association for Computational Linguistics, Dublin, Ireland (Virtual).

Thiago Castro Ferreira, Diego Moussallem, Emiel Krahmer, and Sander Wubben. 2018. Enriching the WebNLG corpus. In Proceedings of the 11th International Conference on Natural Language Generation, pages 171-176, Tilburg University, The Netherlands. Association for Computational Linguistics.

Bhuwan Dhingra, Manaal Faruqui, Ankur Parikh, Ming-Wei Chang, Dipanjan Das, and William Cohen. 2019. Handling divergent reference texts when evaluating table-to-text generation. In Proceedings of the 57th Annual Meeting of the Association for Computational Linguistics, pages 4884-4895, Florence, Italy. Association for Computational Linguistics.

Ondřej Dušek and Zdeněk Kasner. 2020. Evaluating semantic accuracy of data-to-text generation with natural language inference. In Proceedings of the 13th International Conference on Natural Language Generation, pages 131-137, Dublin, Ireland. Association for Computational Linguistics.

Claire Gardent, Anastasia Shimorina, Shashi Narayan, and Laura Perez-Beltrachini. 2017. The WebNLG challenge: Generating text from RDF data. In Proceedings of the 10th International Conference on Natural Language Generation, pages 124-133, Santiago de Compostela, Spain. Association for Computational Linguistics.

Sebastian Gehrmann, Falcon Dai, Henry Elder, and Alexander Rush. 2018. End-to-end content and plan selection for data-to-text generation. In Proceedings of the 11th International Conference on Natural Language Generation, pages 46-56, Tilburg University, The Netherlands. Association for Computational Linguistics.

Ben Goodrich, Vinay Rao, Peter J. Liu, and Mohammad Saleh. 2019. Assessing the factual accuracy of generated text. In Proceedings of the 25th ACM SIGKDD International Conference on Knowledge Discovery Data Mining, KDD '19, page 166-175, New York, NY, USA. Association for Computing Machinery.

Python library. Dateparser.

Kishore Papineni, Salim Roukos, Todd Ward, and WeiJing Zhu. 2002. Bleu: a method for automatic evaluation of machine translation. In Proceedings of the 40th Annual Meeting of the Association for Computational Linguistics, pages 311-318, Philadelphia, Pennsylvania, USA. Association for Computational Linguistics.

Maja Popović. 2015. chrF: character n-gram F-score for automatic MT evaluation. In Proceedings of the Tenth Workshop on Statistical Machine Translation, pages 392-395, Lisbon, Portugal. Association for Computational Linguistics.

Peng Qi, Yuhao Zhang, Yuhui Zhang, Jason Bolton, and Christopher D. Manning. 2020. Stanza: A python natural language processing toolkit for many human languages. In Proceedings of the 58th Annual Meeting of the Association for Computational Linguistics: System Demonstrations, pages 101108, Online. Association for Computational Linguistics.

Lena Reed, Shereen Oraby, and Marilyn Walker. 2018. Can neural generators for dialogue learn sentence planning and discourse structuring? In Proceedings of the 11th International Conference on Natural Language Generation, pages 284-295, Tilburg University, The Netherlands. Association for Computational Linguistics.

Leonardo F. R. Ribeiro, Yue Zhang, Claire Gardent, and Iryna Gurevych. 2020. Modeling global and local node contexts for text generation from knowledge graphs. Transactions of the Association for Computational Linguistics, 8:589-604.

Thibault Sellam, Dipanjan Das, and Ankur Parikh. 2020. BLEURT: Learning robust metrics for text generation. In Proceedings of the 58th Annual Meeting of the Association for Computational Linguistics, 
pages 7881-7892, Online. Association for Computational Linguistics.

Anastasia Shimorina, Claire Gardent, Shashi Narayan, and Laura Perez-Beltrachini. 2018. WebNLG Challenge: Human Evaluation Results. Technical report, Loria \& Inria Grand Est.

Matthew Snover, Bonnie Dorr, Richard Schwartz, Linnea Micciulla, and John Makhoul. 2006. A study of translation edit rate with targeted human annotation. In In Proceedings of Association for Machine Translation in the Americas, pages 223-231.

Elior Sulem, Omri Abend, and Ari Rappoport. 2020 Semantic structural decomposition for neural machine translation. In Proceedings of the Ninth Joint Conference on Lexical and Computational Semantics, pages 50-57, Barcelona, Spain (Online). Association for Computational Linguistics.

Johannes M. van Hulst, Faegheh Hasibi, Koen Dercksen, Krisztian Balog, and Arjen P. de Vries. 2020. Rel: An entity linker standing on the shoulders of giants. In Proceedings of the 43rd International ACM SIGIR Conference on Research and Development in Information Retrieval, SIGIR '20. ACM.

Sam Wiseman, Stuart Shieber, and Alexander Rush. 2017. Challenges in data-to-document generation. In Proceedings of the 2017 Conference on Empirical Methods in Natural Language Processing, pages 2253-2263, Copenhagen, Denmark. Association for Computational Linguistics.

Tianyi Zhang*, Varsha Kishore*, Felix Wu*, Kilian Q Weinberger, and Yoav Artzi. 2020. Bertscore: Evaluating text generation with bert. In International Conference on Learning Representations. 